\title{
Powering the Next Billion Devices with Iot Security
}

\author{
D. Usha Sree, P. Vidya Sagar, G.K.V. Narasimha Reddy
}

\begin{abstract}
The Internet of Thing (IoT) is the connection of computing devices embedded in everyday devices to enable them to send and retrieve data. The Internet of Things (IoT) Is an ongoing sample that broadens the restriction of the internet to comprise a huge collection of processing devices. Associating many unbiased IoT frameworks via the internet offers severa troubles, with safety being up the the front due to the truth a massive part of the accrued data might be furnished to a big and regularly hard to apprehend corporation of spectators. that allows you to make the net of things attainable, the reconciliation of numerous advances is essential, as an instance, tool-to-tool and virtual bodily frameworks. The manner inside the direction of finding out IoT programs gives new troubles because it does not actually incorporates ordinary take a look at systems and strategies. attempting out an IoT framework is based totally upon its the specific setup, and it likewise needs to think about the system diploma and the system state of affairs. At present, organisation and institute endeavors are focusing on convenience and network tests, for example, mimicking the earth wherein the device is to be implemented, and ensuring records is traded in a comfortable manner. in this paper, we make use of the triumphing model of our IoT degree to perform pressure sorting out of our IoT degree below severa conditions.
\end{abstract}

\section{PRESENTATION}

The underlying idea and utilization of the Internet of Things (IoT) showed up as right on time as the Eighties and wound up well known in past due Nineties [1]. Ongoing improvements in severa great areas, collectively with robotization, a long way off sensor structures, installation frameworks and micro-electromechanical systems (MEMS), has quickened the development of the internet of things (IoT) [2,3]. As of now, IoT applications exist in about each difficulty and are assuming an undeniably huge undertaking in our each day existence [4] (e.G., social coverage frameworks, constructing and home computerization, natural checking, basis the executives, strength the board and transportation frameworks), which has brought on the continuing growth of IoT frameworks. As indicated through the Federal Trade Commission (FTC), the amount of IoT gadgets has just dwarfed the amount of human beings in the art work surroundings [5], and the quantity of far off gadgets related to the internet of things may be round 26 billion thru using 2020 and could pretty dwarf middle element gadgets (cellular phones, drugs and computer structures) [6].

Revised Manuscript Received on August 05, 2019.

D. Usha Sree, Assistant Professors of CSE, Gokaraju Rangaraju Institute of Engineering and Technology, Hyderabad, Telangana, India.(Email: dupakuntlausha@gmail.com)

Dr. P. Vidya Sagar, Associate Professor of CSE, Koneru Lakshmaiah Education Foundation, Vijayawada, A.P, India(E-mail: pvsagar20@gmail.com)

Dr. G.K.V. Narasimha Reddy, Professor of CSE, Srinivasa Ramanujan Institute of Technology, Ananthapuramu, A.P, India.(E-mail: csehod@srit.ac.in) inside the maximum state-of-the-art years, the utilization and development of the Internet of Things (IoT) have end up out to be well-known. IoT is the association of processing gadgets implanted in everyday gadgets to empower them to supply and get better records. "A trouble in IoT may be the whole thing shape an person with a heart display embed, a ranch creature with a biochip transponder, a automobile that has worked in sensors [... $]$ or a few specific feature man-made object that may be alloted an IP address and gave the potential to move information over the device." [7-10] With the dynamic improvement of related devices, interoperability between the character devices begins off evolved to get risky. groups utilize various conventions and facts designs for their gadgets and correspondence with severa devices receives difficult. IoT stages on this manner associate severa IoT devices and dealers and convey them into one famous corporation. The degree now not in truth makes interoperability, it makes better methods to dissect and report the statistics gathered thru the devices. The usage of studies and profound studying calculations lets in the manufacturing of recent leads, which at that thing cause sports activities at the associated gadgets.

trying out IoT frameworks have complexities no longer observed in traditional framework businesses, for instance, task net administrations, because of the heterogeneous and significantly circulated nature of its segments (Reetz et al., 2013). a good way to ensure the right on foot of such complicated frameworks, beneficial checks and execution evaluation must be completed earlier than sending an IoT framework in a technology vicinity. in this kind of layout, the real cooperation with the bodily international want to be watched, uniquely in evaluation to everyday programming locating out techniques (Reetz et al., 2013). The heterogeneous concept of IoT additives requests solid checking out capacities to assure control execution meet the consumer necessities simply as manipulate degree understandings amongst professional co-ops and customers. Discerning et al (Cognizant, 2016) characterized the accompanying forms of trying out that need to be completed inner an IoT organic machine:

- useful trying out, which approves the right usefulness of the IoT software.

- Connectivity trying out, it's far in fee of trying out the some distance flung signal a excellent manner to discern out what happens within the event of powerless association, or whilst there are numerous gadgets trying to impart.

Published By:

Blue Eyes Intelligence Engineering 
- common standard performance finding out, approves the correspondence be implemented in order to find out how many synchronous establishments can be bolstered with the useful resource of the usage of a particular device.

- protection trying out, center in protection, approval and confirmation highlights.

- Compatibility trying out, tests the right usefulness underneath various conventions and arrangements.

- Exploratory testing, likewise referred to as purchaser revel in assessments.

There are a few mechanical evaluations about IoT checking out strategies (Cognizant, 2016)(Bloem, 2016)(RCRWireless, 2016), be that as it can, minimal scholastic artwork has been located about trying out IoT frameworks, and the greater a part of them circulate in execution evaluation(Lunardi et al., 2015)(Thangavel et al., 2014)(Vandikas and Tsiatsis, 2014), IoT asset imitating, and IoT testbeds businesses (Sanchez et al., 2014)(Adjih et al., 2016).

in this paper, we appoint the existing phase of our IoT stage execution depending on SwarmOS concept( Costa, 2015) so one can display off our test method that modified into pursued to cover the huge majority of the fields inside the IoT sorting out vicinity. At ultimate we showcase some checking out aftereffects of our basis within the wake of conveying internal unmarried board registering devices. response time and the system throughput have been predicted sooner or later of the exams and a few ends were received with the aid of way of the processing competencies of the gadgets.

\section{WRITING OVERVIEW}

In 2013, Jayavardhana et al "Internet of Things (IoT): A dream, design additives, and destiny headings". The pervasive discovery enacted with the useful resource of wireless network technologies (WSN) covers severa territories of present day life. This causes it achievable to gauge, to deduce and recognise ecological recommendations, fragile ecologies and commonplace property to city situations. The multiplication of those devices in a correspondence incitation machine makes Internet of Things (IoT), wherein sensors and actuators blend impeccably with the earth spherical us and information is shared crosswise over levels to accumulate an common operational image (COP). because of the persevering with adjustment of an collection of empowering some distance flung upgrades, as an example, RFID labels and coordinated sensors and actuator hubs, IoT has risen up out of its outset and is the following soar in advance innovation in converting the internet into a very covered future internet. As creators glide from www (static internet website online pages) to web2 (interpersonal interplay) to web3 (established computer net), the requirement for onrequest facts using complex herbal questions increments significantly. This exam introduces a cloudcentric imaginative and prescient for the worldwide usage of net of things.

In 2014, Chong Tang, Lixing music, Jagadeesh Balasubramani, Shaoen Wu, Saâd Biaz, Qing Yang, and Honggang Wang famous there paper "Relative investigation on CSMA/CA-primarily based totally Opportunistic
Random get right of entry to for net of things". As in keeping with their paintings wireless correspondence is crucial to Internet of Things (IoT). Carrier sensing multiple access/collision avoidance (CSMA/CA)is a properlyconfirmed an extended way flung arbitrary get right of entry to convention and lets in each hub of identical opportunity in getting to a ways flung channel, which brings approximately equal throughput in lengthy haul paying little thoughts to the channel conditions. To misuse hub diverse range that alludes to the distinction of channel situation amongst hubs, this paper proposes two deft irregular get entry to components: blanketed dispute and divided warfare, to useful resource the hub of the great channel situation. inside the covered dispute, the warfare home windows of all hubs percentage a comparable floor of 0 , yet have diverse better limits upon channel state of affairs. in the sectioned war, the dispute window higher positive of a advanced channel circumstance is littler than the lower superb of a more horrible channel state of affairs; to be particular, their warfare home windows are portioned and not the use of a masking. these calculations are moreover cleaned to provide fleeting reasonableness and abstain from retaining the hubs from horrible channel conditions. The proposed components are investigated, actualized, and assessed on a Linux-based totally definitely virtually proving ground and in the NS3 test device. big close to trials showcase that each smart preparations can altogether improve the gadget execution in throughput, postponement, and jitter over the modern-day CSMA/CA convention. particularly, the protected dispute plan can provide seventy 3. three $\%$ and $37.5 \%$ throughput improvements in the basis based definitely and impromptu arranges, one after the alternative. IoT calls for a success medium access conventions for far flung correspondence. This art work proposes deft arbitrary get right of access to versions to abuse hub assorted range in a protracted way off systems. the ones calculations empower hubs to get to the mutual remote channel counting on their channel situations so the hub on the maximum remarkable viable piece price is desired. To abstain from starving hubs with bad channel conditions, a mild setting apart plan are proposed to keep up worldly reasonableness amongst hubs. With massive investigations on a created Linuxbased proving ground and the NS3 set up check gadget, the proposed clever get proper of entry to conspires basically beautify the gadget execution in throughput, postponement, and jitter, that could provide massive favorable times for assisting destiny IoT packages

The In 2014, John A. Stankovic displayed his paper "Exploration instructions for the net of things". As in line with his work numerous specialised networks are overwhelmingly searching for after studies concern topics that upload to the internet of things (IoT). today, as detecting, incitation, correspondence, and manage turn out to be generally contemporary and pervasive, there may be crucial cover within the ones networks, on occasion from pretty alternate factors of view. extra participation between networks is energized. To provide a premise to talking approximately open research problems in IoT, a dream for

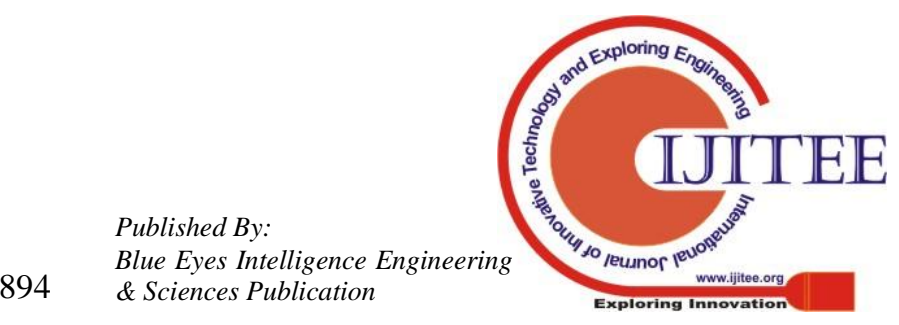


the manner IoT may additionally want to alternate the arena in the inaccessible destiny is first brought. At that element, eight key studies topics are particular and take a look at out issues inner the ones subjects are mentioned. IoT will become an software with elevated complexity in detecting, activation, interchanges, control, and in making analyzing from excellent measures of facts. this can result in subjectively severa tactics of life from these days. What the techniques of existence can be isn't always viable to say. it's far reasonable for country that we can't assume how lives will change. We did now not expect the internet, the net, informal verbal exchange, Face e-book, Twitter, a large variety of applications for PDAs, and so forth., and those have all subjectively modified social orders' way of life. New discover problems emerge due to the big period of devices, the affiliation of the bodily and virtual universes, the receptiveness of the frameworks of frameworks, and intending with problems of safety and protection. it's far depended on that there can be greater collaboration a number of the examination networks so you can address the bunch of issues quicker in reality as to keep away from redesigning the wheel on the same time as a specific community takes care of an difficulty

For In 2015, Hemant Ghayvat, Subhas Mukhopadhyay, Xiang Gui and Nagender Suryadevara proposed their paper "WSNand IOT-primarily based clever houses and Their Extension to smart homes". Their examination technique is to devise and create dependable, powerful, adaptable, realistic, ordinary and much less high-priced nicely being sensor systems for awesome home frameworks The heterogeneous sensor and actuator hubs depending on a ways flung structures management advancements are despatched into the home scenario. those hubs produce ongoing statistics identified with the factor usage and development in the domestic, to estimate the fitness of someone. here, well-being represents how proficiently anyone stays fit inside the home situation and performs out their each day time desk in case you want to hold on with an prolonged and stable lifestyles. We begin the exam with the improvement of the eager domestic method and actualize it in numerous home conditions (various homes) to display display the motion of an occupant for fitness identification. also, our examination stretches out the savvy home framework to clever structures and fashions the plan problems identified with the extremely good shape condition; these form troubles are linked with framework execution and dependability. This exploration paper furthermore examines and represents the possible alleviation to deal with the ISM band obstruction and weakening misfortunes with out buying and selling off best framework execution.

\section{PROTECTION IN IOT}

associated precise reason devices have a critical huge style of capability connection floor territories and verbal exchange designs, which have to all be considered to provide a form to tying down computerized get right of get admission to to to the ones devices. The expression "automatic get to" is utilized right here to apprehend from any obligations which can be helped out via direct tool collaboration wherein get right of access to protection is given via bodily get admission to control. as an example, putting the device proper right into a stay with a lock at the entryway. while bodily get proper of get entry to to can't be denied using programming and machine, measures may be taken to preserve physical access from prompting framework impedance.

As you check out the collaboration designs, see "system manipulate" and "tool records" with a similar diploma of consideration. "system manipulate" may be named any facts that is given to a machine with the useful resource of the usage of any accumulating with the goal of converting or impacting its conduct toward its nation or the state of affairs of its state of affairs. "gadget information" may be named any data that a device emanates to a few unique accumulating about its kingdom and the watched scenario of its condition. so one can enhance protection amazing rehearses, it's far advocated that a humdrum IoT layout is separated into some section/zones as a feature of the hazard displaying exercise. the ones zones are portrayed truely inside the course of this phase and include:

- device,

- problem Gateway,

- Cloud entryways, and

- offerings.

Zones are expansive technique to phase an answer; every region regularly has its private statistics and verification and approval necessities. Zones can likewise be utilized to disconnection harm and confine the effect of low keep in mind zones on higher accept as true with zones.

each location is remoted by using way of a agree with Boundary, that is stated due to the fact the specked red line inside the accompanying chart. It speaks to a trade of records/information beginning with one supply then onto the subsequent. for the duration of this development, the facts/information may be susceptible to Spoofing, Tampering, Repudiation, statistics Disclosure, Denial of provider and Elevation of Privilege (STRIDE). 


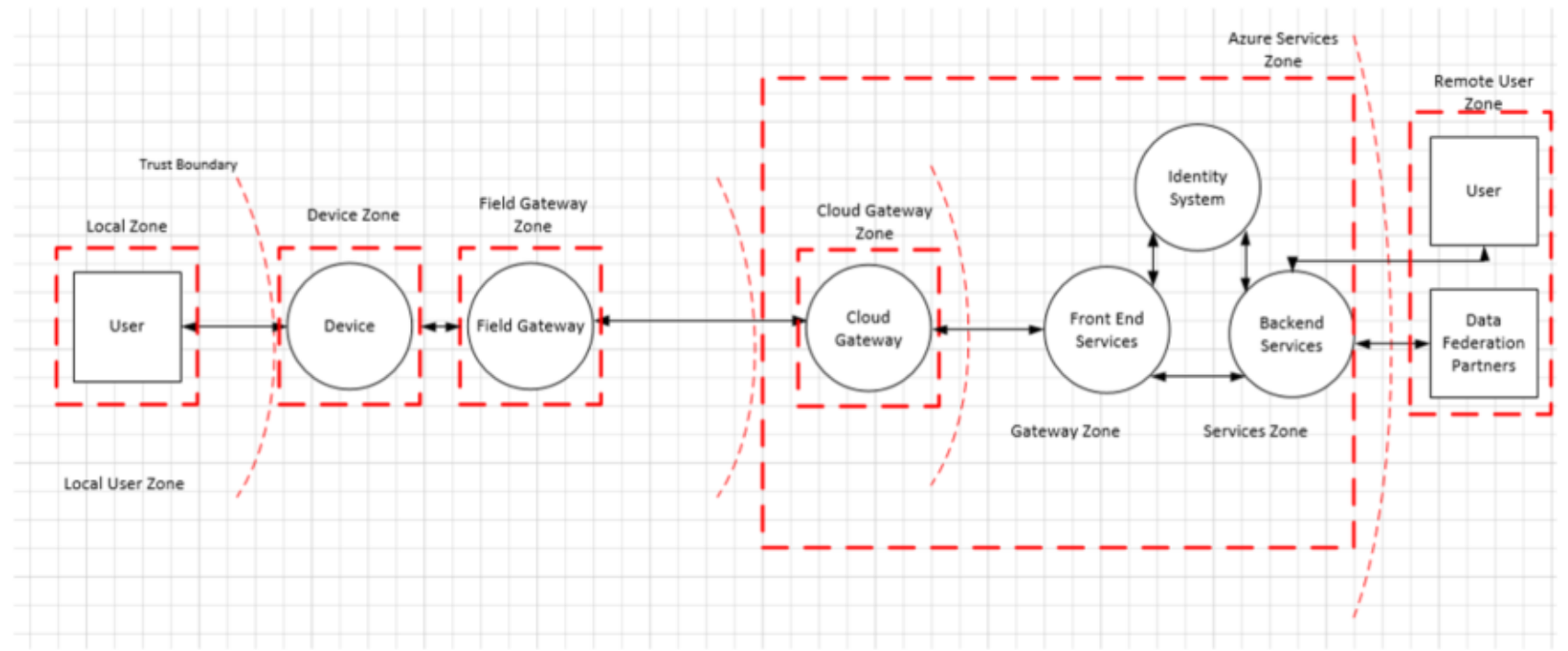

Fig 1 Components of IoT

The segments portrayed interior every limit are likewise exposed to STRIDE, empowering a whole 360 risk demonstrating mind-set on the affiliation. The accompanying areas expound on each one of the elements and particular protection issues and arrangements that ought to be mounted.

The accompanying areas communicate about famous segments at the whole located in the ones zones.

\section{The device place}

The machine situation is the short physical place probable "community installation" shared advanced get entry to to the tool is possible. A "nearby put together" is notion to be a device this is particular and protected from however possibly crossed over to - the open internet, and consists of any short-run a long way flung radio innovation that offers allocated correspondence of gadgets. It does exclude any gadget virtualization innovation making the deception of such a nearby system and it does likewise exclude open administrator organizes that require any gadgets to impart crosswise over open device area if they via hook or by using crook controlled to enter a shared correspondence relationship.

\section{the field door area}

region door is a gadget/system or a few universally beneficial server laptop programming this is going approximately as correspondence empowering effect and, in all likelihood, as a device manipulate framework and system information making ready middle trouble. the sphere entryway region incorporates the sector passage itself and all gadgets which is probably appended to it. because the call infers, concern doorways act outside committed statistics managing offices, are usually place certain, are possibly based upon bodily interruption, and has constrained operational repetition. All to country that a region door is often a element you may contact and harm while comprehending what its functionality is.

A area entryway isn't always much like a minor visitors switch in that it has had a functioning job in overseeing get proper of entry to and statistics flow into, because of this it's throughout the machine in which physical access or

far an utility tended to substance and machine affiliation or consultation terminal. A NAT system or firewall, curiously, could not qualify as area passages given that they may be not unequivocal affiliation or consultation terminals, however alternatively a route (or rectangular) institutions or intervals made via them. The area entryway has unmistakable floor territories. One faces the devices which is probably appended to it and speaks to within the quarter, and one-of-a-type faces every single outer collecting and is the threshold of the area.

\section{The cloud door zone}

Cloud door is a framework that empowers a long way off correspondence from and to gadgets or scenario portals from a few high-quality locations crosswise over open gadget area, frequently within the route of a cloud-based absolutely control and data research framework, a league of such frameworks. occasionally, a cloud entryway can also speedy encourage get proper of access to to precise purpose gadgets from terminals, as an instance, capsules or phones. in the placing pointed out right right here, "cloud" is supposed to allude to a devoted records managing framework that isn't always positive to a similar net website because of the reality the joined gadgets or situation portals. Likewise in a Cloud quarter, operational measures expect centered on bodily access and are not genuinely furnished to an "open cloud" basis.

A cloud entryway can also conceivably be mapped proper into a tool virtualization overlay to defend the cloud passage and most of the people of its related devices or area doors from some other system web website site site visitors. The cloud door itself isn't a tool manipulate framework or a managing or storeroom for tool data; those workplaces interface with the cloud passage. The cloud entryway quarter incorporates the cloud passage itself along all location portals and gadgets straightforwardly or in a roundabout way appended to it. the threshold of the region is a particular ground area wherein each out of doors amassing bring thru. 


\section{The administrations area}

An "manipulate" is characterised for this placing as any product section or module that is interfacing with gadgets thru a area-or cloud entryway for statistics gathering and studies, honestly as for order and manage. Administrations are middle people. They act below their individual inside the route of doors and one-of-a-kind subsystems, keep and spoil down facts, self-sufficiently hassle instructions to devices relying on information testimonies or timetables and locate statistics and manipulate capacities to authorized stop customers.

records devices versus particular purpose gadgets

computers, phones, and tablets are particularly sensible statistics gadgets. phones and drugs are expressly upgraded round growing battery lifetime. They preferably mood killer midway on the same time as not fast taking part with an character, or when no longer giving administrations like playing song or controlling their owner to a selected region. From a frameworks component of view, those facts innovation devices are mainly going about as intermediaries in the direction of people. they may be "people actuators" recommending sports activities and "individuals sensors" amassing input.

specific reason gadgets, from number one temperature sensors to complex enterprise facility advent lines with a large type of segments inner them, are specific. the ones devices are substantially greater perused in reason and regardless of whether or not or now not or not they offer a few UI, they're normally checked to interfacing with or be blanketed into property inside the bodily worldwide. They degree and record herbal situations, turn valves, manage servos, sound indicators, transfer lights, and do severa outstanding errands. They assist to do artwork for which a records gadget is either excessively nonexclusive, excessively high-priced, excessively big, or excessively fragile. The strong cause quick directs their specialized shape too the on hand monetary spending plan for his or her generation and booked lifetime interest. The aggregate of those key components obliges the handy operational strength spending plan, physical impact, and on this manner available potential, figure, and protection capabilities.

at the off danger that a few factor "seems badly" with mechanized or a ways flung controllable gadgets, as an example, physical imperfections or control purpose deformities to decided unapproved interruption and manipulate. The era elements might be overwhelmed, structures might be plundered or burned to the floor, and those is probably harmed or even kick the bucket. that may be a entire awesome beauty of harm than any man or woman maximizing a taken fee card's farthest point. The protection bar for devices that make matters go with the flow, and moreover for sensor records that in the end consequences in tips that purpose topics to move, ought to be better than in any internet based totally organisation or banking scenario.

\section{tool control and machine facts cooperations}

related unique cause devices have a noteworthy shape of capability cooperation floor territories and communication designs, which must all be considered to offer a machine to tying down automatic get right of access to to those devices. The expression "superior get right of entry to" is executed right here to recognize from any duties which can be helped out through direct gadget connection wherein get right of access to safety is given via bodily get entry to manipulate. as an example, setting the machine proper into a stay with a lock at the entryway. whilst bodily access can't be denied using programming and gadget, measures can be taken to keep bodily get right of get admission to to from prompting framework impedance.

As you test out the association designs, see "tool manipulate" and "tool information" with a similar diploma of interest at the same time as hazard displaying. "tool control" can be named any information this is given to a tool by the use of manner of any amassing with the aim of converting or affecting its behavior in the route of its state or the circumstance of its condition. "device statistics" may be delegated any records that a device emanates to 3 different accumulating approximately its u.s. of america and the watched scenario of its state of affairs.

\section{CHECK TECHNIQUE}

finding out the IoT includes the approval method of various viewpoints recognized with tool availability, as an instance, switch velocity, dropped institutions, and so forth. The problems of IoT trying out are beyond programming execution and gadget devices in mild of the reality that IoT offers new multifaceted nature parameters to the exemplary take a look at fashions (Al-Fuqaha et al., 2015). In our unique IoT level we have have been given characterised some types of finding out degrees on the way to approve the whole usefulness of the framework.

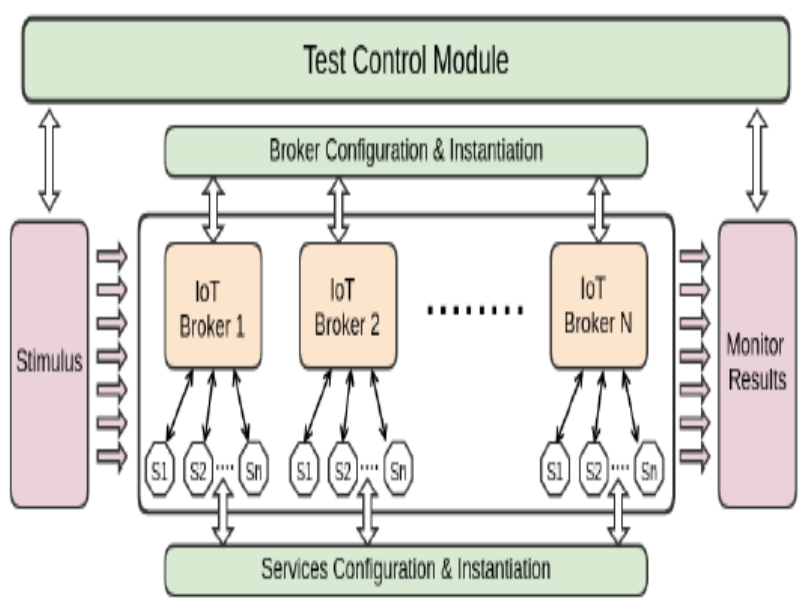

Figure 2: Modular Test Architecture.

Then, there are some important layers that are part of an IoT system and needs to be test. The following Layers and pertaining to test ranges have been characterized for our IoT framework: 
Programming interplay Layer:

- Unitary assessments

- Integration checks

- device checks

- reputation tests

gadget interaction Layer (tool comm and community):

- ordinary general overall performance and Conectivity assessments

- protection checks

- Interoperability tests

customer interplay Layer:

- Usability checks

- Conformance assessments

- Reliability checks

- Scalability tests

\section{EXECUTION TRYING OUT RESULTS}

The execution of our version relies upon on the Java programming language, model 8 . Wharf net server is carried out to have the RESTful administrations inside the IoT set up. For the usage of the assessments in the numerous layers characterized inside the beyond phase we have implemented various devices and systems. Programming interplay Layer: Java code end up produced for the unitary and blend assessments. JUnit Framework coordinated with Maven and Eclipse devices have been applied. For the framework tests, a useful take a look at layout was written in Python language and permits us to mimic the employer module and the administrations. This model serves to carry out each useful check earlier than conveying the framework in express tool devices. useful checks include reputation, discover and vault checks below numerous situations. decide 2 demonstrates our secluded take a look at engineering that has been used to test the usefulness of our software. system interaction Layer (tool comm and community): In request to actualize those layer exams, we've were given characterized some precise experiments for the supply exams. Table1 suggests bandwith experiment wherein standards of check is to alternate the information switch capacity nicely nicely really worth copying a ordinary availability hassle. Table 2 present the scenario even as the gadget is all of a shocking have become off.

Table 1: Bandwitdh tests.

\begin{tabular}{|l|l|}
\hline Purpose & Test Bandwidth during register process \\
\hline Pre-condition & Broker and Service running \\
\hline Criteria & Change the bandwidth \\
\hline Test Procedure & $\begin{array}{l}\text { Service registering in Broker } \\
\text { Increase/decrease bandwidth }\end{array}$ \\
\hline Expected & Delay in the register process. Timeout. \\
\hline
\end{tabular}

With a purpose to achieve performance check of our platform, we have efficaciously deployed our implementation on three special hardware systems which might be presented in desk 1 . To test our application in a actual surroundings and under pressure situations, we use a load technology device known as Tsung, which may be configured to decide the wide sort of request in line with $2 \mathrm{~d}$
$100 \mathrm{Req} / \mathrm{sec}$

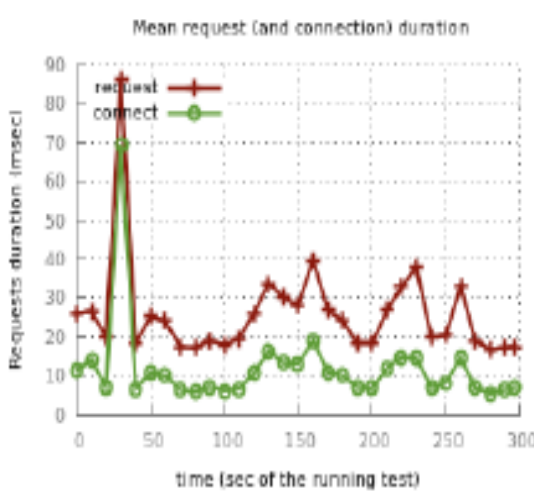

$200 \mathrm{Req} / \mathrm{sec}$

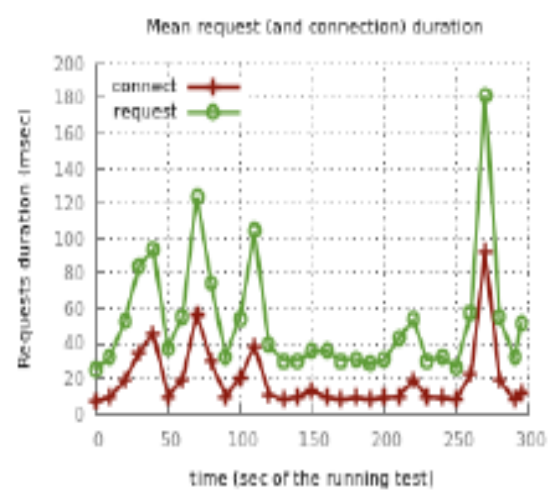

$300 \operatorname{Req} s / \operatorname{Sec}$

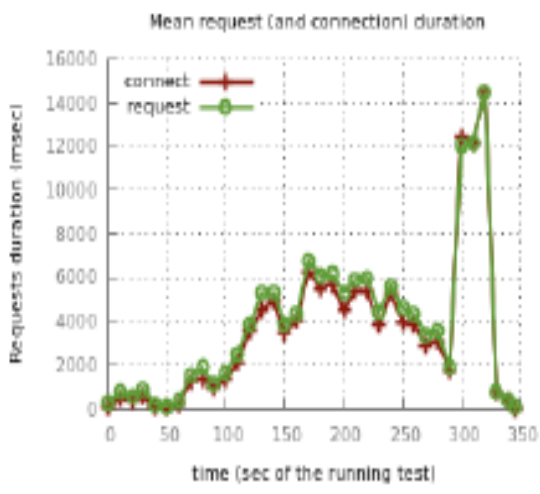

Figure 3: Results for 100, 200 and 300 requests/sec in Intel Edison device

Table 2: Connectivity tests - Switched-off.

\begin{tabular}{|l|l|}
\hline Purpose & Broker switched-off suddenly \\
\hline Pre-condition & Broker and Service running \\
\hline Criteria & Switch-off broker \\
\hline Test Procedure & $\begin{array}{l}\text { Service registering in Broker } \\
\text { Switch-off the broker suddenly }\end{array}$ \\
\hline Expected & Broker maintain the registered services \\
\hline
\end{tabular}

\section{Extension results}

Effect on Wi-Fi clients Po WiFi is designed to provide high cumulative channel occupancies for power delivery while minimizing the effect on Wi-Fi traffic. To evaluate this, we deploy a PoWiFi router and evaluate its effect on Wi-Fi traffic. We use a Dell Inspiron 1525 laptop with an Atheros chipset as a client associated with our router on channel 1. We compare four different schemes: - Baseline. PoWiFi is disabled on the router, that is, the router introduces no extra traffic on any of its interfaces. BlindUDP. The router transmits UDP broadcast traffic at $1 \mathrm{Mbps}$ so as to maximize its channel occupancy. PoWiFi. The router sends UDP broadcast traffic at $54 \mathrm{Mbps}$ and uses the queue threshold check in Section 3.1. - NoQueue. The router sends UDP broadcast traffic at $54 \mathrm{Mbps}$ but disables the queue threshold check. We evaluate PoWiFi with various Wi-Fi traffic patterns and metrics: the throughput of 
UDP and TCP download traffic, the page load time (PLT) of the 10 most popular websites in the United States, 1 and traffic on other Wi-Fi networks in the vicinity of our benchmarking network. Effect on UDP traffic. UDP is a common transport protocol used in media applications such as video streaming. We run iperf with UDP traffic to a client $7 \mathrm{ft}$ from the router. The client sets its Wi-Fi bitrate to $54 \mathrm{Mbps}$ and runs five sequential copies of iperf, $3 \mathrm{~s}$ apart. We repeat the experiments with target UDP data rates between 1 and 50Mbps, and measure the achieved throughput computed over $500 \mathrm{~ms}$ intervals. All the experiments are run during a busy weekday at UW CSE, with multiple other clients and 43 other Wi-Fi networks operating at $2.4 \mathrm{GHz}$. Figure $5 \mathrm{a}$ plots the average UDP throughput as a function of the 11 tested UDP data rates. The figure shows that Blind UDP significantly reduces throughput. With No Queue, the router's kernel does not prioritize the client's iperf traffic over the power traffic. This results in roughly a halving of the iperf traffic's data rate as the wireless interface is equally shared between the two flows. With PoWiFi, however, the client's iperf traffic achieves roughly the same rate as the baseline. This result demonstrates that PoWiFi effectively prioritizes client traffic above its power traffic. For the PoWiFi experiments above, Figure $6 \mathrm{a}$ plots the CDFs of individual channel occupancies on the three Wi-Fi channels. The figure shows that the individual channel occupancies are around 5-50\% across the channels. The mean cumulative occupancy, on the other hand, is $97.6 \%$, demonstrating that PoWiFi can efficiently deliver power even in the presence of UDP download traffic.

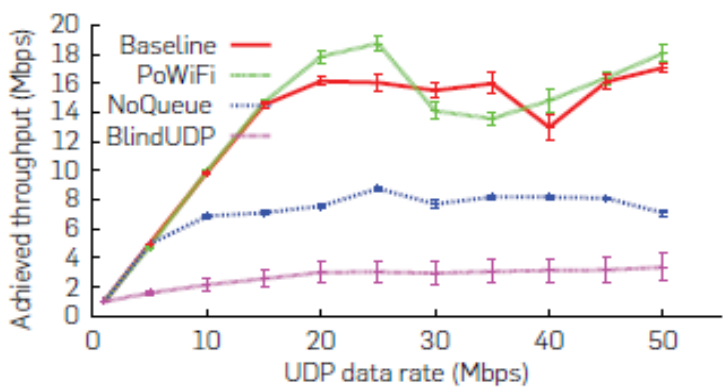

(a)

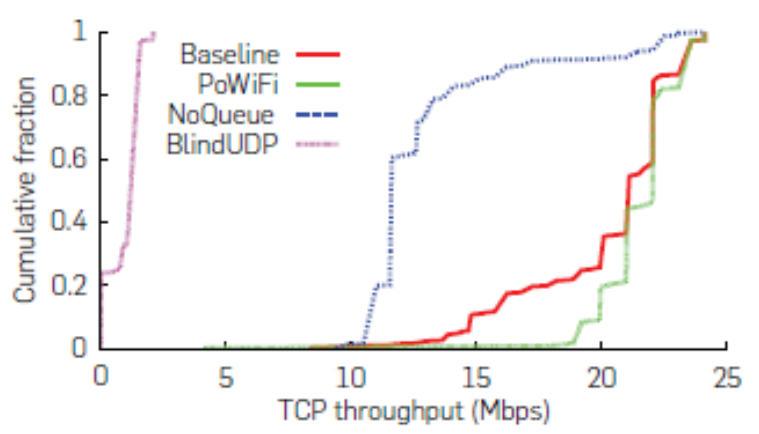

(b)

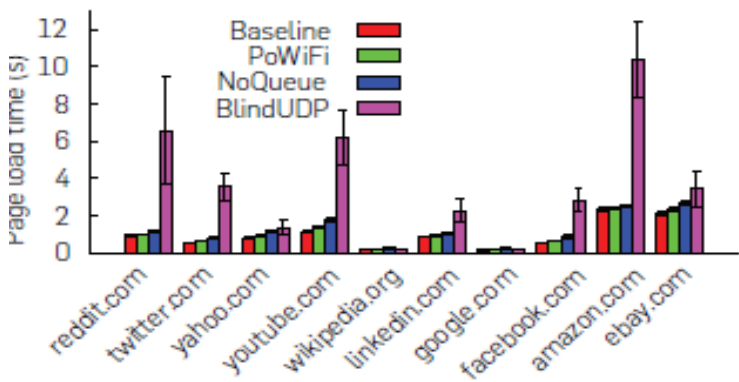

(c)

Effect on Wi-Fi traffic. The figures show the effect of various schemes on TCP and UDP throughput as well as the page load times

of the top 10 websites in the United States.1 The plots show that PoWiFi minimizes its effect on the Wi-Fi traffic. (a) UDP experiments, (b) TCP experiments, and (c) PLT experiments

\section{CONCLUSION AND FUTURE WORK}

An ever increasing quantity of ranges increase and strive to drag inside the customer with their examination and AI capacities. due to a lacking which means that of the segments internal an IoT diploma, this paper have furnished. additionally, this artwork introduces the underlying trying out aftereffects of our IoT utility. we've got were given characterised 3 extremely good layers for attempting out: programming, device and client. For our future research, we intend to maintain with the strain sorting out but under diverse situations, as an example increasing and diminishing the intensity of the wifi sign of the gadgets. safety exams need to be actualized because as of now we're executing the confirmation modules of our foundation.

\section{REFERENCES}

1 WhatIs.Com, "net of factors (IoT)," IoT time desk, july sixteen. [Online]. accessible: http://internetofthingsagenda.Techtarget.Com/definition/i nternet-of-subjects-IoT. [Accessed: 23-Apr-2017].

2 J. Guth, U. Breitenbucher, M. Falkenthal, F. Leymann, and L. Reinfurt, "exam of IoT level models: A subject pay interest relying on a reference engineering," 2016, pp. 1-6.

3 P. Scully, "IoT Analytics five topics To recognize approximately The IoT Platform environment," 26-Jan2016. [Online]. accessible: https://iot-analytics.Com/fivetopics-knowabout-iot-diploma/. [Accessed: 09-May2017].

4 Hong Kong Polytechnic university, "person and get admission to control (IAM)." [Online]. to be had: https://www.Polyu.Edu.Hk/ags/e-e-e-

newsletter/news0911/I AM_details.Html. [Accessed: 23Apr-2017].

5 Symantec safety response, "IoT gadgets being gradually implemented for DDoS attacks," Symantec safety response, 22-Sep-2016. [Online]. on hand: http://www.Symantec.Com/associate/web journals/iotdevicesbeing-progressively carried out ddos-assaults. [Accessed: 09-May-2017]. 
6 Gartner Says the net of factors installed Base Will growth to 26 Billion gadgets through 2020; Gartner Inc.: Stamford, CT, americaA., 2013.

7 MobilityFirst future net shape. to be had at the internet: http://mobilityfirst.Winlab.Rutgers.Edu/(had been given to on 19 June 2017).

8 EXpressive net shape. available at the internet: https://www.Cs.Cmu.Edu/ xia/(were given to on 19 June 2017).

9 Named statistics Networking. available at the net: http://named-facts.net/(were given to on 19 June 2017).

10 Cloud destiny net shape project. available on the internet: http://cloud fia.Org (had been given to on 19 June 2017). 